International Journal of Engineering \& Technology, $7(2.7)(2018)$ 33-36
International Journal of Engineering \& Technology
WPC
Website www.sciencepubco.com/index.php/IJET
Research Paper

\title{
Smart farming: disease detection in crops
}

\author{
G. Balram*1 ${ }^{*}$ K. Kiran Kumar \\ ${ }^{1}$ Research Scholar, Department of CSE, KLEF, Vaddeswaram, A.P. \\ ${ }^{2}$ Professor, Department of ECSE, KLEF, Vaddeswaram, A.P. \\ *Email: balaramcse@cvsr.ac.in
}

\begin{abstract}
Automation in the market has been for decades now in the market. These systems run all through the day but there has not been any design which regards to identifying and classifying objects in specific and giving apt solution to them. Latest technologies like image processing and advanced machine learning mechanisms have become the core of the science and innovations.

Our project's idea is a broad concept that integrates various subjects and generates solutions to energy saving, cooling, automation and structural design. To get the desired results, above are the few constraints which play a major role in yielding high productivity from the crops. And besides them, the classification of objects through image processing and machine learning is used instead of the man power to accomplish the target to reduce the amount of hardware used.
\end{abstract}

Keywords: Agriculture; Automation; Classification; Disease; Image Processing.

\section{Introduction}

Agriculture is the backbone for the human sustenance. India is well known for its agriculture production. Most of population is dependent on agriculture. Farmers have variety of options to cultivate crops in the field. Still then, cultivating these crops for best harvest and top quality of production is done in a technical way. So the yield can be increased and quality can be improved by the use of technology. To increase the rate of productivity, automation has been introduced into this field. Besides automating, there are also few problems to be attended which need solutions.

This is the point where we have identified few constraints like disease detection and the intervention of the weed plants inhibits the plant growth which are absolutely necessary for the plant growth and so they can be automatically identified without manual power and the disease detection in the plants, is implemented in our project through machine learning and image processing.

Generally, whenever there is disease to a plant, we can say that leaves are the main indicators of the disease caused to the plant. Mostly, we can see the spots on the leaves of it due to disease. However when the amount of disease to the plant is large then the whole leaf gets covered by the disease spots. Detecting this at an earlier stage might help in preventing the spread of the disease to the entire crop.

The growth of weed plants inhibits the growth of the crop as the weed plant absorbs all the nutrition present in the soil. Hence, their removal is necessary for the healthy growth of the plants. Hence, these factors served to be the needs and the importance of taking up this project.

\section{Key Aspects}

To increase the rate of productivity, automation has been introduced into this field. Besides automating there are also few problems to be attended which need solutions.

This is the point where we have identified few constraints like Temperature, Humidity, Light, Water which are absolutely necessary for the plant growth. Besides this, the intervention of the weed plants inhibits the plant growth and so they can be automatically removed without manual power and this is implemented in our project through machine learning and image processing.

Leaf miners are the insect family at larval stage .They feed between upper and lower part of the leaf. Due to this, it is severely damaged. On a single leaf the number of maggots can be six. Therefore, it can severely damage the plant. It can restrict plant growth, which leads to reduced yields. Hence, we can develop a technique using image processing to detect the disease, and classify it. This will avoid human interference and hence lead to précised unprejudiced decision.

Generally, whatever our observation is, about the disease , it is just used for deciding the type of the disease. Disease symptoms on a plant are the visible effects. They can be change in color, shape or functional changes of the plant as per its response to the pathogens, insects etc.

Automation is already an important aspect of the agro based efficiency techniques. Introducing such technologies and mechanisms can bring a major change in the traditional systems. Because this device helps us in greatly reducing the cost of manual effort and maintenance, this becomes economical. When Machine Learning comes into the scenario, the capabilities of the system turns to be 
endless and our project is one such innovation and has greater possibilities for further more implementations.

\section{Usage of Technologies}

Introducing the automation technologies, all the above mentioned features and mechanisms can bring about a major change in the traditional systems. Because this device helps us in greatly reducing the cost of manual effort and maintenance, this is extremely economical.

Generally, whenever there is disease to a plant, we can say that leaves are the main indicators of the disease caused to the plant Mostly, we can see the spots on the leaves of it due to disease. However when the amount of disease to the plant is large then the whole leaf gets covered by the disease spots. Detecting this at an earlier stage might help in preventing the spread of the disease to the entire crop.

The growth of weed plants inhibits the growth of the crop as the weed plant absorbs all the nutrition present in the soil. Hence, their removal is necessary for the healthy growth of the plants.

Being aware of the soil nutrition is important because, if adequate minerals are not present in the soil, this may deteriorate the growth of the plants. Hence, the owner should be aware of the nutrition content present in the soil.

When Image processing and Machine Learning comes into the picture, the capabilities of the system turns to be endless and our project is one such innovation and has greater possibilities for further more implementations. This greatly reduces the manual power both in efforts and prediction for the crop.

The device is capable of providing farmers with information about all kinds of possible activities and changes that are taking place in the crop field. Since we are integrating both the automation techniques, pest identification and the removal of weed plants, this stands out unique in the market and results in the greater market influence.

Once a disease is developed in plants, it spreads very fast and multiplies the rate of spreading the disease at a larger rate. Once it affects, it will reduce the quantity \& quality of the crop, it reduces the rate process of photosynthesis. So, the disease detection plays an important role in the field of agriculture. By using the proposed system, though we could not prevent the disease completely, it can at least reduce the rate at which the disease has infected the plants.

The pests and infections in the crop are usually identified by the farmers and the pesticide is sprayed manually, but by the time they identify it, the disease spreads all over the crop. This device detects the pest infestation at the start itself.

In this system, we have used Raspberry pi module for detecting the disease as it has image processing capabilities. As soon as disease is detected, the message is transmitted to another module of the device. Then proper measures can be taken appropriately automatically on infected area by using the trained system. The farmers need not check each leaf manually. Prevention is always better than cure is the motive behind introducing the proposed system. Instead of taking measures for the affected area after the disease is attacked, we can prevent it at the start itself.

The device module detects the above constraints of the soil and treats them respectively with their requirements.

The following are the primary concerns for introducing the new system:

\section{B. To reduce manual power}

\section{Generating data for future predictions}

\section{Image Processing and Machine Learning}

Usage of computer algorithms on digitalised images to perform image processing is referred to as the Digital Image Processing. An image is defined as a two-dimensional function $f(x, y)$, where $\mathrm{x}$ and $\mathrm{y}$ are spatial coordinates. The amplitude of any pair of coordinates namely, $(\mathrm{x}, \mathrm{y})$ is referred to as the intensity or the grey level of the given image at that point. When $\mathrm{x}, \mathrm{y}$ and the intensity values of $f$ are all finite and discrete quantities, we refer the image as a digital image.

A digital image is composed of a finite number of elements and each has a particular location and value. These elements are referred to as picture elements, image elements and pixels respectively. The most widely used term to denote the elements of an image is a pixel. Images play an important role in the human perception because, vision is the most advanced sense that humans are gifted with. To create process, communicate and display digital images, the digital image processing has been in the use since past few years. So in brief, the input is the image, it undergoes image processing using few of the efficient algorithms and finally gives the image as the output.

The following is the algorithm used to perform the above functionality:

- The image is captured in RGB format.

- The color transformation structures are generated.

- The color values are converted from RGB to the space specified in the structure.

- Image segmentation is performed by applying K-means clustering algorithm.

- The green pixels are masked.(Masking green channel).

- The masked cells that are present inside the edges of the infected cluster are eliminated.

- The infected cluster is converted from RGB to HIS.

- SGDM matrix is generated for $\mathrm{H}$ and $\mathrm{S}$.

- GLCM function is called to calculate its features.

- Texture statistics are computed.

- For recognition, k-nn (classifier) is configured.

\section{Working}

1) Login/Register: The user has to create an account and then login with his credentials to ensure security. If the credentials don't match, he will not be shown the user interface.

2) Activation of camera: The camera is started and the images of the plants are clicked. The camera slides along the XY-slot table. This facilitates the clicking of pictures along the whole field.

3) Image Processing: The images are uploaded into the database. Then the image processing is performed on the images of the leaves to ensure there is no disturbance in the image. By making use of the existing data sets, the clicked images are compared and the disease is detected.

4) Notification: When the disease is detected, the farmer is alerted through a notification and preventive measures. 


\section{Tools and Framework}

The proposed device does not need huge hardware appliances to become a powerful one. The Raspberry pi, camera module, XYslot table and few sensors are the only hardware requirement that the project needs. All the rest can be achieved just with the software technologies. The major software requirements are the image processing libraries, Raspbian Jessie operating system and an application for the device control. Since, this is a cost effective product, every average income based individual can purchase it. So, any software technologies can be made use of for the further enhancements in the project and different implementations can be done.

The following is the technology stack which is made use to implement the above functionality:

\section{A. OpenCV}

OpenCV stands for Open Source Computer Vision. It is machine learning software library. It contains a library of programming functions. It is required for real time applications that are related to image processing. OpenCV is available in $\mathrm{C}, \mathrm{C}++$ languages and its major interface is in $\mathrm{C}++$. However, it still retains a less widespread though it has very wide interface with $\mathrm{C}$ language.

\section{B. Python}

Python is modest and easy to learn. The programming related to Raspberry PI is performed using python. It supports all kinds of modules and packages. It also has an interpreter along with a standard library. They are available in both source as well as binary form. They are free to all platforms, and can be freely distributed to all. Python is a scripting language, that means it allows to execute the code line by line.

\section{Tomcat server}

There are basically two kinds of servers.

1. The Application server,

2. The Web server

The Tomcat server is an open source web server that was developed by the Apache Software Foundation. In our system, the database consists of the images of the infected and healthy leaves that have been clicked from various angles. This data base is large. Also for image processing, some processes need java based systems. So we use this Tomcat server.

The following are the images which show how the plants are affected and how the image processing helps us in resolving the problem of identifying the disease without the use of manual power:

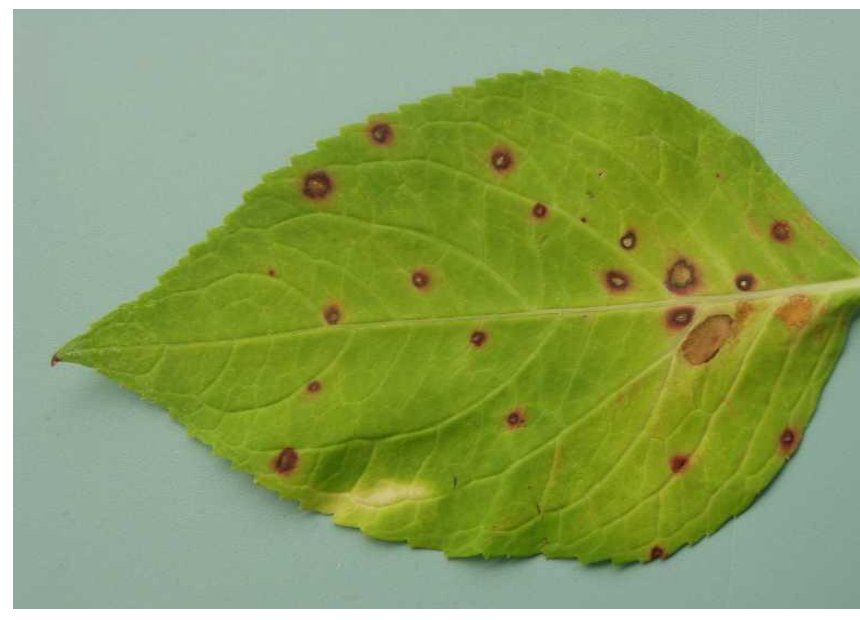

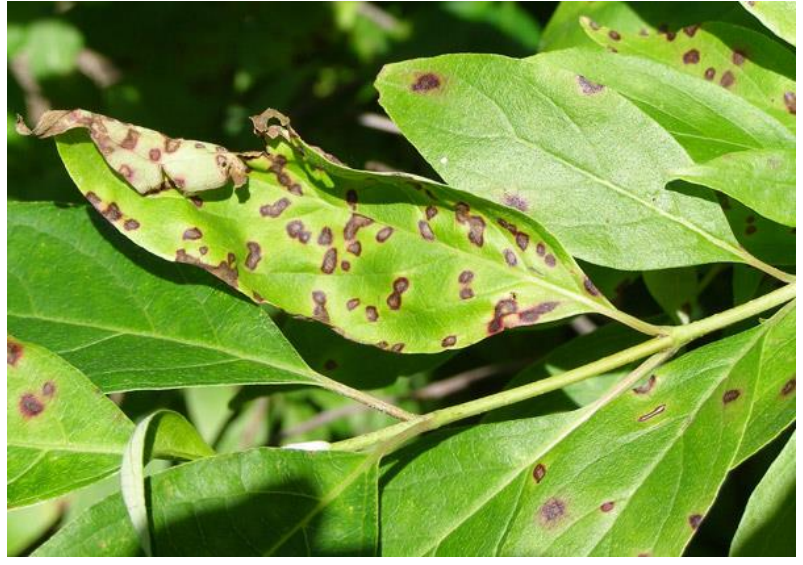
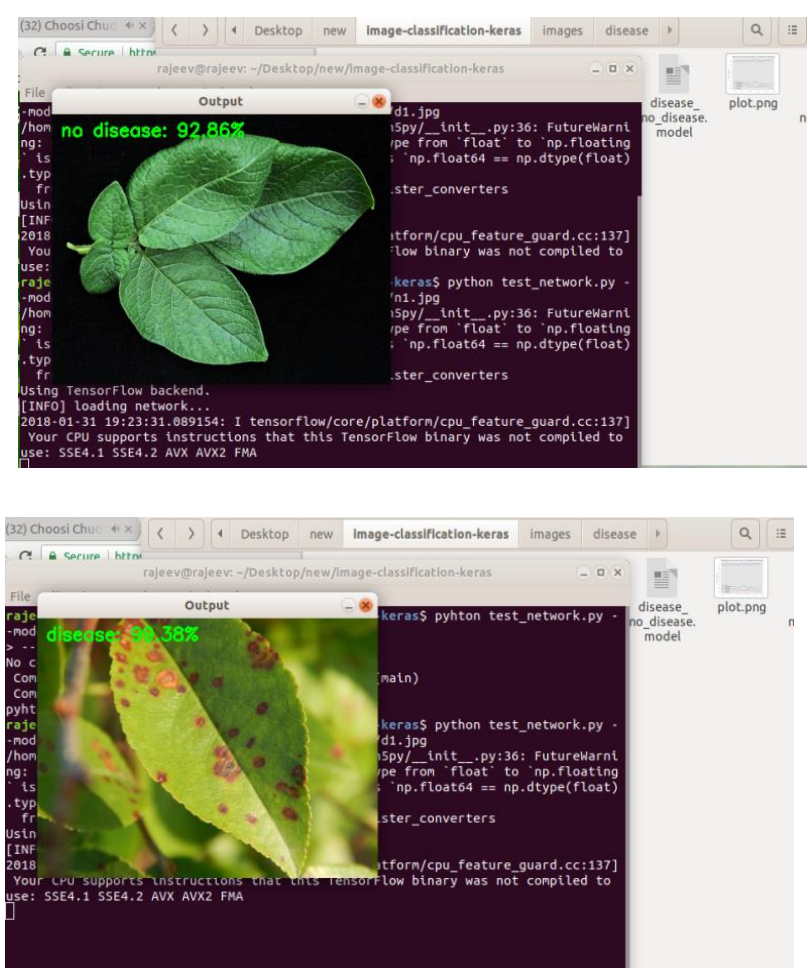

\section{Scope of the device}

These devices can widely be used in any scenario. Once a consumer based version is developed, these devices can be trained to match the consumer needs. These can also be used in farms to keep watch of the creatures that may lead to the destruction of the crop. It also is not very costly. All it requires is the piece of code to be embedded and an enhancement in the couple of hardware appliances.

Since smart farming is a concept quickly getting its upper hand on agriculture by offering high-precision crop control, useful data collection for analysis cum future use, and automated farming techniques, there are clearly many advantages for the fate of farming.

The device is capable of providing farmers with the information regarding crop yields, pest attacks, and soil nutrition etc which can be used to improve farming techniques over time. Since we are integrating both the automation techniques, pest identification and the removal of weed plants, this stands out unique in the market and results in the greater market influence.

In brief, the following are the services provided by the proposed device:

- $\quad$ Addressing the constraints like Temperature, $\mathrm{Hu}$ midity, Light, and Water by automation.

- Inhibiting the growth of weed plants. 
- Detecting diseases at earlier stages through image processing.

- Generating data for future predictions.

- Pest identification.

- Analyzing the data generated for future predictions.

\section{Conclusion}

This idea is a broad concept that integrates various subjects and generates solutions to energy saving, cooling, automation and structural design. To get the desired results, above are the few constraints which play a major role in yielding high productivity from the crops. And besides them, the classification of objects through image processing and machine learning is used instead of the man power to accomplish the target to reduce the amount of hardware used.

\section{References}

[1] http://www.ijcsmc.com/docs/papers/May2016/V5I5201682.pdf

[2] http://www.ijcsmc.com/docs/papers/February2017/V6I2201719. pdf

[3] http://ieeexplore.ieee.org/document/7155951/

[4] https://apsjournals.apsnet.org/doi/full/10.1094/PDIS-03-150340-FE

[5] https://www.sciencedirect.com/science/article/pii/S22143173163 00154

[6] https://ac.els-cdn.com/S1474667017455613/1-s2.0S1474667017455613-main.pdf?_tid=d3e71a40-041a-11e8-8e6c00000aacb35d\&acdnat $=1517137571 \quad 5 \mathrm{e} 74 \mathrm{~d} 03172 \mathrm{e} 0 \mathrm{~d} 329 \mathrm{ca} 2615$ $4796789 \mathrm{da} 9$

[7] https://www.hindawi.com/journals/cin/2016/3289801/

[8] https://github.com/marshallr3/Crop-Disease-Diagnosis

[9] https://www.frontiersin.org/articles/10.3389/fpls.2016.01419/full

[10] M. Reyalat, Al Hairy, Bani Ahmad, Braik and Z.Rahamneh, "Fast and Accurate Detection and Classification of Plant Diseases".

[11] Smith J S., Camargo A., "An image processing based algorithm to automatically identify plant disease visual symptoms".

[12] https://www.researchgate.net/publication/266079421_Advanced _methods_of plant disease detection_A review 